\title{
Traduire
}

Revue française de la traduction

$220 \mid 2009$

Organisations internationales | Bicentenaire de Louis Braille

\section{Le rôle du traducteur à la Cour européenne des droits de l'homme}

James Brannan

\section{CpenEdition}

Journals

Édition électronique

URL : http://journals.openedition.org/traduire/378

DOI : $10.4000 /$ traduire.378

ISSN : 2272-9992

Éditeur

Société française des traducteurs

Édition imprimée

Date de publication : 15 juin 2009

Pagination : 24-35

ISSN : 0395-773X

Référence électronique

James Brannan, «Le rôle du traducteur à la Cour européenne des droits de l'homme », Traduire [En ligne], 220 | 2009, mis en ligne le 12 novembre 2013, consulté le 19 avril 2019. URL : http:// journals.openedition.org/traduire/378; DOI : 10.4000/traduire.378 


\title{
Le rôle du traducteur à la Cour européenne des droits de l'homme(1)
}

\author{
James Brannan
}

La Cour européenne des droits de l'homme, qui siège à Strasbourg, fête cette année ses 50 ans. Elle fut créée au sein du Conseil de l'Europe en 1959 afin de contrôler le respect de la Convention européenne des droits de l'homme de 1950(2). Si les tâches et méthodes du traducteur ont beaucoup évolué au fil des années, sa raison d'être reste la même : le fonctionnement de la Cour dans ses deux langues officielles, le français et l'anglais. Un juge de la Cour souligne "le rôle important " de la traduction juridique dans son travail quotidien (Popovic 2006 : p. 386). Ceci étant, l'activité de la Cour a connu une augmentation exponentielle de son activité depuis 50 ans, sans croissance corrélative de l'effectif linguistique. Aujourd'hui, les 800 millions d'habitants des 47 États membres du Conseil de l'Europe peuvent déposer une requête devant la Cour. Sa jurisprudence a un immense impact dans toute l'Europe ; elle conduit les États à modifier leur législation et constitue une référence pour les juridictions internes. Le nombre d'arrêts et de décisions rendus par la Cour en 2008 s'élève à près de 31500 . II est intéressant de comparer ce volume de travail avec, d'une part, celui de la Cour internationale de Justice (CIJ) à La Haye, qui en 2008 a rendu trois arrêts et deux ordonnances d'importance dans des affaires purement interétatiques, et de l'autre, celui de la Cour de justice des Communautés européennes (CJCE) à Luxembourg, dont le nombre d'arrêts et d'ordonnances rendus est de 550 à 600 par an. La Cour européenne des droits de l'homme, tout en maintenant le bilinguisme comme principe de base, a dû faire des choix face aux impératifs économiques, car il est devenu impossible de tout traduire. La traduction systématique dépend donc de l'importance de l'affaire, et ce sont les juristes du greffe eux-mêmes qui traduisent généralement à partir des langues non officielles. Le rôle du traducteur a ainsi évolué, s'agissant du type de documents à traduire ou à vérifier et des délais à respecter. Le greffe de la Cour compte aujourd'hui

(1) Les opinions exprimées dans cet article n'engagent que son auteur. Remerciements à Emmanuel Simonet de la division française pour sa relecture du texte.

(2) Ci-après " la Convention ". 
dix-sept postes de traducteur (dont quatre ayant le statut de "réviseur ") dans ses deux divisions linguistiques (française et anglaise), chacun traduisant vers sa langue maternelle(3).

La Cour n'a pas de "Statut ", à la différence d'autres juridictions internationales, mais les grandes lignes de son organisation sont énoncées dans la Convention elle-même et dans son Règlement. Alors que le principe général du régime linguistique de la $\mathrm{ClJ}$ est consacré par l'article 39 de son Statut, il faut se rapporter au Règlement de la Cour de Strasbourg pour trouver une indication identique (article 34) : "Les langues officielles de la Cour sont le français et l'anglais ". L'ordre des langues est inversé dans la version anglaise: The official languages of the Court shall be English and French(4). En outre, l'article $76 \S 1$ du Règlement prévoit :

La Cour rend tous ses arrêts en français ou en anglais, sauf si elle décide de rendre un arrêt dans les deux langues officielles.(5)

Jusqu'en novembre 1998, tous les arrêts étaient rendus dans les deux langues officielles. Avant l'entrée en vigueur, cette année-là, du Protocole $n^{\circ} 11$ instituant la " nouvelle " Cour, fusionnant ainsi le travail de l'ancienne Cour et celui de la Commission européenne des droits de l'homme, organe de filtrage, le nombre d'affaires renvoyées par la Commission devant la Cour était minime par rapport au volume traité aujourd'hui. L'article 76 permet à la Cour de rendre des arrêts exceptionnellement dans les deux langues - c'est le cas principalement des affaires de Grande Chambre - mais la vaste majorité des affaires ne donne pas lieu à un texte bilingue. En revanche, lorsque la publication des arrêts dans le recueil officiel est décidée, l'article $76 \S 2$ prévoit l'usage des deux langues officielles de la Cour. II existe une disposition similaire (l'article 57) pour les " décisions " (concernant la recevabilité, ou plus rarement la radiation, d'une requête) $)^{(6)}$ et, comme nous allons le voir, de ces deux articles du règlement de la Cour dépend une partie importante de l'activité du traducteur.

D'autres juridictions internationales, ayant par ailleurs un effectif équivalent de traducteurs, ont adopté des régimes linguistiques similaires. Malgré l'existence de six langues officielles au sein des Nations Unies aujourd'hui, la ClJ n'a retenu que les deux langues de son prédécesseur, le français et l'anglais( (7). Cela peut se comprendre pour les raisons évoquées dès 1920 : le multi-

(3) À ne pas confondre avec le service de traduction générale du Conseil de l'Europe (voir les articles de Jean-François Allain dans Traduire $\mathrm{n}^{\text {os }} 214$ et 215), dont l'effectif est environ le double ; en outre, les interprètes qui travaillent lors des audiences et délibérations de la Cour ne font pas partie du personnel du greffe.

(4) Alors que la version anglaise du statut de la CIJ maintient le français devant l'anglais, reflétant, dit-on, le fait que le français est toujours la plus importante des deux langues!

(5) C'est nous qui soulignons.

(6) La Cour peut rendre également des avis consultatifs, ce qu'elle n'a fait qu'une seule fois, en 2008.

(7) Le prédécesseur de la ClJ, la Cour permanente de Justice internationale, avait déjà adopté, dans son statut de 1921, le français et l'anglais comme ses deux langues officielles, mais les délégués anglophones au Conseil de la Société des Nations ont dû se battre pour assurer à l'anglais le même statut que le français ! Voir Brannan 2004 ; la ClJ a 17 postes de traducteur au total (mai 2009), voir http://www.icj-cij.org/registry/fr/nav2_1.pdf 
linguisme au sein d'une juridiction internationale risque de diminuer l'autorité de la chose jugée. Et si une seule version linguistique est désignée comme faisant foi, comment choisir ? Ensuite il y a la difficulté purement matérielle - la nécessité d'embaucher des traducteurs. Le Tribunal pénal international pour l'ex-Yougoslavie (TPIY), autre juridiction onusienne, n'a que le français et l'anglais comme langues officielles et de travail, malgré l'importance des langues de l'ex-Yougoslavie ${ }^{(8)}$. La nouvelle Cour pénale internationale (CPI) a également adopté l'anglais et le français comme langues principales, mais son statut prévoit l'usage des quatre autres langues des Nations Unies comme langues officielles supplémentaires (notamment pour la publication future de sa jurisprudence) et comme langues de travail ad hod ${ }^{(9)}$.

Un régime très différent en la matière est celui de la Cour de justice des Communautés européennes (CICE) ${ }^{(10)}$ à Luxembourg (l'autre Cour européenne !), avec un personnel linguistique qui constitue presque la moitié de son effectif total : la direction de la traduction compte environ 900 collaborateurs, dont 600 sont des traducteurs (appelés juristes-linguistes)(11). La CJCE, comme les autres institutions de l'Union européenne, garantit une parité linguistique ${ }^{(12)}$ entre les États membres, avec un total de 23 langues actuellement ${ }^{(13)}$. II s'agit d'un régime linguistique qui ne serait évidemment pas réaliste dans une juridiction mondiale, mais qui est donc possible, sous réserve d'un budget adéquat dans une juridiction dite "régionale " comme la CICE. Le principe du multilinguisme en droit communautaire est souvent défendu comme étant un moyen de garantir l'égalité des citoyens devant la loi et la sécurité juridique (Despretz 2003 : p. 87). Ce régime fait néanmoins l'objet de critiques. Olivier Moréteau, en proposant que l'anglais devienne la langue commune du droit européen, le décrit comme un gâchis, surtout financier, mais aussi comme une illusion, " car l'égalité des citoyens devant la loi n'est pas pour autant respectée " (Moréteau 1999 : p. 158-59), dans la mesure où une simple traduction ne reflète pas toujours les réalités juridiques nationales. Force est de constater, cependant, que la CJCE a maintenu le français comme langue de travail unique, notamment pour ses délibérations, malgré la « pression » récente de certains juges qui préfèrent l'anglais et le fait que l'anglais est davantage parlé que le français dans les nouveaux États membres (Duparc-Portier 2007 : p. 1059, et Terris 2007 : p. 78-9). En outre, le service de traduction de la CJCE a dû entériner un système de traduction par relais (ou langue " pivot ") pour certaines combinaisons

(8) Les juges eux-mêmes ne viennent pas des pays de l'ex-Yougoslavie ; pour cette juridiction voir Sirois 2000.

(9) Article 50 du Statut de la CPI ; en mai 2009 son service de traduction compte environ 15 fonctionnaires (traduisant vers le français pour la plupart).

(10) Voir Duparc-Portier (2007), Despretz (2003), Berteloot (2000), Gallo (1999) ; nos références à la CJCE comprennent aussi le Tribunal de première instance.

(11) Chiffres communiqués à l'auteur en avril 2009.

(12) La diversité linguistique est désormais garantie par la Charte des droits fondamentaux de l'Union européenne (article 22).

(13) Une des langues est adoptée comme la langue de la procédure et les arrêts de la Cour doivent être publiés dans l'ensemble des langues officielles de l'Union. 
linguistiques ${ }^{(14)}$. Cette solution avait auparavant été écartée comme se heurtant à deux règles : celle de l'autonomie de chaque unité linguistique et " celle de la traduction directe, qui seule peut garantir un maximum d'adhésion au texte original et un niveau de précision conforme à celui qu'on exige d'une traduction juridique " (Gallo 1999 : p. 79).

La Cour européenne des droits de l'homme est aussi une juridiction internationale dite "régionale ", couvrant néanmoins une plus grande variété de langues potentielles (37) avec ses 47 États. Cependant elle n'a que deux langues officielles, qui sont de fait aussi ses « langues de travail ", bien que cette dernière expression ne soit pas utilisée dans le Règlement de la Cour. Ce choix s'imposait à la création de la Cour, car le français et l'anglais étaient déjà les deux langues officielles de sa maison mère, le Conseil de l'Europe ${ }^{(15)}$, créé en 1949. Ce sont également les langues de son acte fondateur, la Convention européenne des droits de l'homme, dont les deux textes font également foi. Lors de l'institution de la " nouvelle "Cour en 1998, il était légitime de se demander si on ne devait pas ajouter le russe comme langue officielle, suite à l'entrée au Conseil de l'Europe des pays d'Europe centrale et orientale. Une traduction en russe de la jurisprudence de la Cour aurait contribué à sa diffusion dans les milieux juridiques de ces pays. Mais pour citer Giorgio Malinverni :

Si on s'était avisé de proposer une troisième langue officielle, d'autres grands États n'auraient certainement pas manqué cette occasion pour réclamer que la liste soit alors allongée au profit de leur langue nationale. (Malinverni 2000 : p. 543).

Le régime linguistique de la "nouvelle " Cour a néanmoins été aménagé en faveur des justiciables. Les requérants peuvent utiliser leur propre langue dans certaines conditions, notamment pour leur requête et, sous réserve d'autorisation, pour leurs observations orales ou écrites. Dans ce cas, "le greffier prend les dispositions nécessaires en vue de l'interprétation ou de la traduction, intégrale ou partielle, en français ou en anglais des observations orales ou écrites du requérant " (article $34 \S 3$ du Règlement). Les États parties aux procédures devant la Cour, pour leur part, doivent utiliser les langues officielles ou bien faire traduire leurs observations à leurs frais. La politique de la Cour reflète sa propre jurisprudence dans la mesure où la Convention reconnaît, en l'occurrence dans le cadre de procédures pénales, le droit de "se faire assister gratuitement d'un interprète ". La langue ne doit pas constituer un obstacle à l'accès effectif à la justice. II serait parfois trop onéreux pour un requérant de faire traduire ses observations à ses propres frais ou de payer un interprète lors d'une audience. Par contre, les gouvernements peuvent plus facilement se faire assister par des juristes maîtrisant l'anglais ou le français. La faculté d'utiliser des langues non officielles a néanmoins fait l'objet de critiques en raison des frais de traduction dont on pourrait faire l'économie (Lambert 2002 : p. 800), mais en réalité, comme nous allons le voir, dans la vaste majorité des affaires les pièces en question ne sont pas confiées à un traducteur.

(14) Par exemple, l'allemand est la langue " pivot " pour la langue bulgare.

(15) Son Assemblée parlementaire utilise également l'allemand, l'italien et le russe comme "langues de travail ", voir Duparc-Portier (2007) : p. 1054. 
Le traducteur n'intervient pas, de façon générale, au stade de la requête. Les requêtes sont attribuées aux juristes du greffe en fonction de leur connaissance de la langue et du système juridique concerné. Les juristes commencent alors à rédiger l'exposé des faits, en français ou en anglais, généralement sans l'assistance d'un traducteur. Si les pièces du dossier sont déposées dans une langue non officielle, le juriste compétent traduit lui-même les éléments essentiels pour la partie "En fait ». II arrive aussi qu'il demande à un traducteur connaissant sa langue de l'aider à traduire, par exemple, des dispositions du droit interne ou des propos litigieux dans une affaire concernant la liberté d'expression. Le juge Popovic, dans un article sur le droit comparé à la Cour, rappelle l'importance de cette activité quotidienne de traduction à ce stade de la procédure, quoique souvent en dehors des services linguistiques :

Cette opération délicate, qui est d'une importance énorme pour les travaux de la Cour, [...] se répète chaque jour dans des dizaines de cas, et cela en partant de langues très diverses. Les juristes, collaborateurs de la Cour, ainsi que les juges, y sont parfaitement habitués. (Popovic $2006:$ p. 373)

Les juristes de la Cour doivent pouvoir maîtriser au moins une des langues officielles (comme les juges), mais à moins d'être francophones ou anglophones ils rédigent donc dans une langue qui n'est pas leur langue maternelle. Ce qui explique l'existence d'un " garde-fou " : le contrôle linguistique (language checking) par un locuteur natif. II s'agit d'un service (devenu quasi obligatoire pour les décisions et arrêts les plus importants) qui est mis à la disposition des juristes depuis quelques années, assuré à la fois par des traducteurs et par une équipe de correcteurs linguistiques (dont les premiers ont été recrutés en 2007). Dès lors que les textes des décisions ou arrêts sont destinés à être publiés, ne serait-ce que sur le site Internet de la Cour, un tel contrôle est bien sûr essentiel. Les juges qui siègent dans une affaire donnée ne sont pas non plus forcément francophones ou anglophones, et leurs opinions individuelles (concordantes ou divergentes) peuvent également faire l'objet d'un tel contrôle. Le processus ainsi mis en place s'est avéré très efficace, bien qu'il prolonge légèrement, normalement d'une semaine ou deux, la durée du traitement des requêtes. C'est dans ce contexte que le traducteur ou le correcteur linguistique peut également donner son avis au juriste ou au juge sur une amélioration potentielle du texte source ou sur une question linguistique d'ordre général. Des fichiers d'erreurs récurrentes, qui pourront être consultés par les juristes du greffe, sont en préparation dans chaque division linguistique. La correction linguistique donne lieu à un échange entre le linguiste et le juriste qui est enrichissant pour les deux parties : le linguiste est ainsi sensibilisé aux spécificités des différents systèmes juridiques représentés à la Cour et peut proposer une solution adaptée à une notion donnée, en anglais ou en français, même s'il ne parle pas la langue source. Faute d'avoir un service conséquent de juristes-linguistes capables de faire face à toutes les combinaisons linguistiques, il s'agit d'un compromis néanmoins fiable. Au niveau de la traduction d'une langue officielle à une autre, le fait que le texte source peut résulter d'une telle traduction « noire », pour citer Weston (2005 : p. 447), peut nécessiter une vigilance accrue. Ce n'est peut-être pas une situation idéale, mais même à la CJCE, comme nous l'avons vu, la traduction n'est plus forcément " directe " - les compromis sont parfois inévitables. 
Le rôle du traducteur est plus étendu au stade initial de la procédure lorsqu'il s'agit d'une affaire devant la Grande Chambre de dix-sept juges (et non pas une chambre de sept juges). Le traitement bilingue de ces affaires ressemble davantage au système de l'ancienne Cour, avant novembre 1998, ou à celui de la ClJ actuellement. La Grande Chambre peut être saisie de deux manières : à la suite d'un renvoi à la demande d'une partie (demande accueillie dans dix affaires en 2008), ou lorsqu'une chambre se dessaisit d'une affaire (cinq en 2008) ; dans les deux cas il s'agit d'une procédure exceptionnelle, justifiée principalement par l'intérêt jurisprudentiel. Un traducteur du greffe est affecté à l'affaire, de bout en bout, pour la traduction d'une langue officielle à l'autre, en fonction de la langue dans laquelle le projet d'arrêt sera rédigé. Un autre traducteur sera désigné, le cas échéant, pour la correction linguistique du texte source. Quant aux pièces du dossier (observations des parties, textes législatifs, décisions des juridictions internes, etc.) qui doivent en principe exister dans les deux langues officielles, leur traduction, quelle que soit la langue dans laquelle elles ont été déposées, est souvent soustraitée. Le traducteur interne s'occupe, dans des délais plutôt serrés, des documents hautement confidentiels à destination des juges, en vue de l'audience et des délibérations. Ces documents comprennent la note du rapporteur, les questions aux parties et parfois un rapport du service de recherche du greffe. Le traducteur assiste aux délibérations, à l'issue desquelles les juges votent sur le dispositif de l'arrêt. II peut être amené à apporter des suggestions linguistiques, voire appelé à défendre sa traduction du projet d'arrêt. Après les délibérations, il continue à collaborer avec les juristes et les juges pour peaufiner le texte définitif de l'arrêt, ainsi que pour traduire les opinions individuelles des juges, le cas échéant. II faut noter que le texte de l'arrêt de Grande Chambre fait foi dans les deux langues officielles. Le traducteur aura en fin de compte une responsabilité plus grande, ne serait-ce qu'à cause de la valeur juridique de sa traduction, ce qui explique l'importance de son rôle dans les affaires de Grande Chambre.

À la différence de certaines juridictions internationales, comme la CIJ, la Cour de Strasbourg ne fait plus traduire les comptes rendus d'audience (la CJCE a également abandonné cette pratique - voir Despretz 2003 : p. 90) ; depuis peu, les audiences (avec l'interprétation dans les langues officielles) sont retransmises en différé sur le site Internet de la Cour. Mais comme ailleurs, en plus des arrêts et décisions, les traducteurs à Strasbourg doivent traduire les documents internes que le greffe prépare dans l'une de ses langues officielles - procès-verbaux de réunions, rapports jurisprudentiels, informations administratives et pratiques diverses destinées au personnel - ainsi que les autres documents publics. II s'agit notamment des communiqués de presse (à traduire dans des délais très courts), des notes d'information jurisprudentielle, des rapports annuels, des discours occasionnels, et des annonces pour le site Internet. Le document public le plus important est certainement le recueil officiel des décisions et des arrêts. Le Comité des publications sélectionne les affaires méritant de figurer dans le recueil, publié dans les deux langues officielles. Martin Weston (ancien réviseur à la Cour) a regretté le fait que ces textes ne soient pas tous traduits en amont (Weston 2005 : pp. 451-52). II suggère que les traducteurs sont encore plus motivés quand l'affaire est en cours et souligne 
la " valeur ajoutée " du traducteur lorsqu'il repère des corrections à apporter au texte source, au stade du projet. II est vrai que le système actuel génère une quantité de traductions en souffrance parfois difficile à gérer face à d'autres impératifs ; la CJCE avait d'ailleurs décidé à un moment donné de traduire sa jurisprudence a posteriori mais, devant l'effet pervers de cette décision, est revenue à la traduction en amont. II serait, en outre, préférable d'offrir au public un accès immédiat à la jurisprudence dans les deux langues officielles. Cependant, avec la charge de travail existante des divisions linguistiques et à cause du délai supplémentaire qui serait occasionné par une traduction non strictement nécessaire à ce stade, un tel " traitement bilingue " reste limité à Strasbourg. La vaste majorité des textes du recueil est donc traduite après que l'arrêt ou la décision ont été rendus, sauf pour les affaires de Grande Chambre. Le traducteur pourra toujours indiquer des corrections mineures éventuelles à apporter au texte source, car le Règlement prévoit la rectification d'erreurs a posteriori par la Cour. Le service des publications, d'ailleurs, exerce un dernier contrôle sur tous les textes destinés au recueil. Par ailleurs, le traducteur peut consulter le dossier de l'affaire et interroger le juriste compétent, même si l'affaire n'est plus en cours. Dès que la traduction est finie, sans attendre la publication du recueil, elle sera disponible sur " Hudoc "(16). Certains arrêts sont publiés sous forme d'extraits, si l'intérêt jurisprudentiel réside dans une partie seulement. Par ailleurs, un sommaire est rédigé (et traduit) spécialement pour chaque affaire. Enfin, contrairement à la pratique d'autres juridictions internationales, les deux versions linguistiques ne sont plus publiées " en regard "(17), un format qui serait pourtant utile pour les traducteurs ! A l'ère de l'Internet il est même légitime de se demander s'il est toujours nécessaire de publier un recueil, mais la Cour y tient jusqu'à présent, notamment pour des raisons de prestige ; toutes les juridictions internationales ont une telle publication.

II est bien sûr souhaitable que la jurisprudence de la Cour touche le maximum de lecteurs parmi les 800 millions d'Européens, et le fait de ne publier que dans deux langues officielles peut paraître insuffisante. II est surtout regrettable que les arrêts ne soient pas traduits systématiquement dans la langue du requérant (voir Terris 2007 : p. 75, et Malinverni 2000 : p. 547). Il a même été suggéré que la Cour gagnerait à consacrer son budget de traduction à la publication de ses arrêts dans la langue du pays concerné plutôt que dans son autre langue officielle (voir Krüger : p. 164). Le rapport récent du "Groupe des Sages " (adressé au Comité des ministres du Conseil de l'Europe) a souligné que les institutions judiciaires et administratives nationales devraient être en mesure d'avoir accès à la jurisprudence importante de la Cour dans leurs langues respectives afin de faciliter l'identification des arrêts susceptibles d'être pertinents pour résoudre les affaires qui leur sont soumises. Mais la Cour de Strasbourg, comme nous l'avons constaté, n'est pas à même, pour des raisons d'impératifs économiques, d'assurer la même égalité linguistique que la Cour de Luxembourg. Dans son avis sur le

(16) La base jurisprudentielle en ligne (en français et en anglais), http://www.echr.coe.int/echr/fr/hudoc/

(17) Cette pratique a été abandonnée en 1998 ; la CIJ est restée attachée à ce principe. 
rapport des "Sages ", elle a donc indiqué que les gouvernements concernés seraient obligés de prendre en charge ce travail de traduction vers les langues non officielles. La Cour a également constaté :

Certains États traduisent et publient les arrêts de la Cour dans leur journal officiel, mais ils ne le font généralement que pour les affaires dirigées contre eux-mêmes. Or les juridictions de tout État contractant doivent avoir connaissance aussi bien des arrêts rendus contre l'État en question que des grands arrêts prononcés par la Cour [... $]^{(18)}$

À défaut de traduire l'intégralité d'un arrêt, il s'agirait d'en produire un résumé, sur support papier et/ou électronique. Le gouvernement allemand a déjà pris l'initiative de faire traduire dans sa langue certains arrêts contre l'Allemagne ${ }^{(19)}$. II est évident, par ailleurs, que les traducteurs et les correcteurs linguistiques de la Cour ne doivent jamais oublier la possibilité que leurs textes soient retraduits vers des langues tierces. La clarté du texte sera d'autant plus importante ; il faut éviter toute expression qui pourrait induire en erreur un traducteur externe.

Le traducteur a aussi un rôle à jouer dans l'organisation de la terminologie " maison ", en l'absence d'un service de terminologie propre à la Cour (il en existe un, par contre, au sein du service de traduction générale du Conseil de l'Europe). La tâche d'organiser des réunions de terminologie, plusieurs fois par an, est confiée à un traducteur de chaque division linguistique, qui prépare une liste aux fins de la discussion, à laquelle participent parfois des juristes du greffe. L'ordre du jour comporte souvent des termes des langues non officielles pour lesquels il s'agit de trouver une traduction standardisée. Un des projets actuels est de constituer une base de données en utilisant le logiciel Multiterm de Trados, et de la mettre à la disposition du personnel du greffe. Les traducteurs ont également la tâche d'alimenter et de mettre à jour les guides de rédaction et de style spécifiques à la Cour.

II relève aussi du rôle du traducteur de veiller à la sécurité juridique des arrêts par la cohérence de ses choix terminologiques. II ne s'agit pas seulement de retrouver les citations de la jurisprudence mais de vérifier s'il existe une traduction " consacrée " d'une expression juridique particulière. Le cas échéant, le traducteur doit se tenir aux "précédents linguistiques " (Weston 2005 : p. 458), car s'il omet de reprendre un terme déjà utilisé dans un contexte donné, le lecteur risque de ne pas faire le rapprochement avec la jurisprudence existante - ce qui peut être d'autant plus grave si le lecteur est une autorité nationale, comme par exemple la Chambre des Lords, qui est souvent amenée à interpréter la jurisprudence de la Cour. Dans une affaire récente, les Lords ont examiné l'adjectif anglais flagrant figurant dans l'expression flagrant

(18) " Avis de la Cour sur le rapport des sages (tel qu'adopté lors de la session plénière du 2 avril 2007) ", page 3 ; voir le lien à http://www.echr.coe.int/ECHR/FR/Header/Reports+and+Statistics/Reports/Other+reports/ (consulté en mai 2009).

(19) http://www.coe.int/T/D/Menschenrechtsgerichtshof/Dokumente_auf_Deutsch/ (voir Krüger 2007 : p. 164). 
denial of justice telle qu'utilisée par la Cour dans le contexte des risques de violation de l'article 6 de la Convention lors d'une expulsion vers un État tiers ${ }^{(20)}$. II s'agissait en l'espèce d'une traduction de l'expression française " déni de justice flagrant ", utilisée depuis l'arrêt Soering c. Royaume-Uni de 1989 dans lequel l'expression anglaise était, par contre, flagrant denial of a fair trial. II semblerait que, depuis Soering, l'anglais ait été " aligné " sur le français pour exprimer une notion plus large, mais l'adjectif en question est resté le même dans les deux langues. Dans un autre arrêt de 2009, la Chambre des Lords a même tiré des conclusions d'une structure grammaticale utilisée par la Cour : a rigorous scrutiny must necessarily be conducted $^{(21)}$. Par ailleurs, on ne peut qu'approuver le choix du traducteur français de la Cour, en l'espèce, de maintenir l'ambiguïté du passif par la formule " il faut impérativement soumettre à un contrôle attentif ... n. Le traducteur ne doit pas oublier qu'une expression donnée, qu'elle vienne de la Convention ou de la jurisprudence, pourrait faire l'objet d'une interprétation par une juridiction interne ; il ne faut pas qu'il s'écarte des précédents et il doit éviter d'introduire des interprétations personnelles. Le risque de " traductions multiples " face à des " citations implicites " a été signalé également à la CICE (Despretz 2003 : p. 94). Dans son travail quotidien, le traducteur se réfère donc à maintes reprises à la base Hudoc et utilise la fonctionnalité " concordance " de Trados. La recherche dans ce corpus jurisprudentiel sera plus difficile si les " précédents linguistiques " n'ont pas toujours été respectés. Bien entendu, les traducteurs se familiarisent avec les citations " cachées " et le " jargon " interne au fil du temps.

II faut savoir, en outre, que dans sa jurisprudence la Cour utilise un certain nombre de termes qui ont des sens dits " autonomes ", provenant principalement de la Convention. II s'agit de notions que la Cour détache du contexte juridique national pour les doter d'un sens " européen " valable pour tous les États contractants. Par ce biais, elle assure l'indispensable uniformité d'interprétation de la Convention et une certaine égalité devant la loi, en évitant que les normes protectrices des droits de l'homme ne varient selon les qualifications propres aux droits nationaux. Sudre (1998: p. 96-98) énumère les concepts formellement qualifiés par la Cour d'" autonomes " : arrestation, droits et obligations de caractère civil, matière pénale, témoins, accusation, peine, biens. Il ajoute que d'autres concepts sont présentés comme " autonomes " dans la doctrine : aliéné, autorité judicaire, condamnation, contestation, correspondance, détention, domicile, loi, magistrat, partie lésée, privation de liberté, tribunal, vagabond, victime, vie familiale. Le traducteur se voit obligé de choisir un tel terme, dans la partie "En droit " des arrêts de la Cour, même s'il ne serait pas forcément approprié en droit interne. Le terme " accusé " au sens de l'article 6 de la Convention est un bon exemple. En effet, il pourrait sembler surprenant pour un traducteur français de qualifier une personne d'" accusé " pendant l'enquête préliminaire voire au stade de la garde à vue, mais c'est le terme qui convient dans ce contexte jurisprudentiel. Pour la Cour, " l'accusation " peut se définir " comme la notification

(20) RB (Algeria), etc, [2009] UKHL 10, 18 février 2009.

(21) Secretary of State for the Home Department v Nasseri, [2009] UKHL 23, 6 mai 2009, § 15, citant l'arrêt Jabari c. Turquie, $\mathrm{n}^{\circ}$ 40035/98, § 39, CEDH 2000-VIII. 
officielle, émanant de l'autorité compétente, du reproche d'avoir accompli une infraction pénale ", et dans certaines affaires ${ }^{(22)}$ le requérant a donc été considéré comme un "accusé " dès sa garde à vue. La CJCE a également créé sa terminologie propre (voir Berteloot 2000 : p. 530) afin d'exprimer des notions particulières de droit communautaire, comme par exemple " effet direct ", expression composée de deux termes courants ayant acquis un sens spécifique.

La base de données Hudoc est une ressource facile d'accès pour les confrères traducteurs qui s'intéressent à la terminologie de la Cour. Comme nous l'avons vu, si la date de l'arrêt ou la décision est postérieure à novembre 1998, il n'existera en général de traduction, en anglais ou en français, que dans les affaires de Grande Chambre ou lorsque le texte aura été sélectionné pour publication au recueil. II existe néanmoins quelques traductions exceptionnelles, notamment si l'affaire a un grand intérêt public ou médiatique, comme dans le cas de la décision d'irrecevabilité Al Fayed c. France du 27 septembre 2007 qui intéressait beaucoup le public britannique! Pour repérer la version originale des arrêts de chambre (depuis 1998), on peut se référer à la mention qui figure à la fin du dispositif : " fait en anglais " ou " fait en français ". Les arrêts de Grande Chambre, rendus dans les deux langues, n'ont pas juridiquement de " version originale " et ils ne comportent aucune indication de la langue du projet d'arrêt. Cependant, celle-ci peut parfois être devinée selon l'État défendeur en question : un arrêt contre un État francophone, ainsi qu'un petit nombre d'autres États, aura été rédigé au départ en français ${ }^{(23)}$. L'anglais étant plus souvent la langue originelle de rédaction que le français, il y a davantage de traductions vers le français que vers l'anglais. Les opinions individuelles des juges comportent l'indication " traduction " le cas échéant. La base Hudoc constitue ainsi un outil pour les traducteurs extérieurs, et pas seulement ceux qui travaillent en anglais et en français, car on retrouve souvent des termes provenant de langues tierces, laissés entre parenthèses, et des passages entiers de droit interne traduits directement ou indirectement des langues non officielles. On peut dire que le rôle du traducteur à Strasbourg est aussi d'apporter son savoir-faire à l'élaboration d'une ressource fiable de terminologie, à laquelle les traducteurs juridiques européens pourront ensuite se référer(24).

Certes, les besoins linguistiques de la Cour européenne des droits de l'homme, comme de toute juridiction internationale, peuvent créer certains problèmes d'organisation. Pour citer un

(22) Voir, par exemple, Subiali c. France, $n^{\circ}$ 65372/01, § 46, 14 septembre 2004, et Salduz c. Turquie [GC], $n^{\circ} 36391 / 02, \S 54,27$ novembre 2008.

(23) Les juristes de certains autres pays, notamment l'Italie, l'Espagne, le Portugal, la Grèce et, dans une proportion non négligeable, la Turquie et la Roumanie, ont également tendance à rédiger en français plutôt qu'en anglais.

(24) Des lexiques juridiques publiés par le Conseil de l'Europe, et dont la terminologie émane en grande partie de la jurisprudence de la Cour, sont connus depuis un certain nombre d'années déjà, notamment celui de Frank Bridge (The Council of Europe French-English Legal Dictionary de 1994). 
juge de la Cour, Language issues bedevil all international courts(25) (Terris 2007 : p. 71). Mais les juges sont aussi les premiers à reconnaître le rôle indispensable des traducteurs et autres linguistes du greffe, qui sont bien intégrés à l'activité judiciaire. Les traducteurs travaillent en étroite collaboration avec les juges, les juristes et les services de publication et de presse, et sont reconnus comme faisant partie intégrante du processus d'élaboration des textes.

James.Brannan@echr.coe.int

Originaire de Londres, James Brannan est diplômé en langues et en droit (diplôme de droit comparé et $D E A$ en droit communautaire) et il exerce le métier de traducteur depuis presque 20 ans. Après quelques années de salariat, il s'est mis à son compte, travaillant dans le domaine juridique à Lyon, où il a été nommé expert judiciaire en 1998. Il a assuré plusieurs formations spécialisées, y compris pour la SFT, dont il est membre depuis 2001. Il a pris ses fonctions actuelles de traducteur à la Cour européenne des droits de l'homme (Strasbourg) en 2005, après trois ans à la Cour internationale de justice (La Haye).

\section{Bibliographie}

BERTELOOT, P., " La traduction juridique dans I'Union Européenne, en particulier à la Cour de Justice " in La traduction juridique : Histoire, théorie(s) et pratique, ASTTI/ETI, 2000, p. 521-535 (disponible à www.tradulex.org/).

BRANNAN, J., "Translation at the World Court: the Weight of History ", American Translators Association Chronicle, vol. XXXIII, $\mathrm{n}^{\circ}$ 10, 2004, p. 38-42.

DESPRETZ, M., "La traduction juridique à la Cour de Justice des Communautés européennes " in GREENSTEIN (dir.), Langue, culture et code : regards croisés, Paris, L'Harmattan, 2003, p. 85-97.

DUPARC-PORTIER, P., "La question des langues en Europe : entre paradoxes et divergences juridiques ", Revue trimestrielle des droits de l'homme, $n^{\circ}$ 72, 2007, p. 1051-1085.

GALLO, G., "Les juristes linguistes de la Cour de justice des Communautés européennes ", in SACCO et CASTELLANI (dir.), Les multiples langues du droit européen uniforme, Turin, L'Harmattan Italia, 1999, p. 71-88.

(25) "Les questions linguistiques tourmentent toutes les juridictions internationales!". 
KRÜGER, H.-C., "The importance of protecting human rights on the national level " in Trente ans de droit européen des droits de l'homme, HARTIG (éd.), Bruxelles, Bruylant, 2007, p. 151-166.

LAMBERT, P., "Quelle réforme pour la Cour européenne des droits de l'homme ? ", Revue trimestrielle des droits de l'homme, $\mathrm{n}^{\circ}$ 52, 2002, p. 791-805.

MALINVERNI, G., "Le régime linguistique de la procédure devant la nouvelle Cour européenne des droits de l'homme ", in Les droits de l'homme au seuil du troisième millénaire, Bruxelles, Bruylant, 2000, p. 541-548.

MORETEAU, 0., "L'anglais pourrait-il devenir la langue juridique commune en Europe " in SACCO et CASTELLANI (dir.), Les multiples langues du droit européen uniforme, Turin, L'Harmattan Italia, 1999, p. 143-162.

POPOVIC, D., " Le droit comparé dans l'accomplissement des tâches de la Cour européenne des Droits de l'Homme " in Liber amicorum Luzius Wildhaber : Droits de l'homme, regards de Strasbourg, CAFLISCH et al. (éd.), Kehl, Engel, 2007, p. 371-386.

SIROIS, A., "La traduction et l'interprétation devant les tribunaux pénaux internationaux " in La traduction juridique : Histoire, théorie(s) et pratique, ASTTI/ETI, 2000, p. 537-557 (disponible à www.tradulex.org/).

SUDRE, F., "Le recours aux "notions autonomes" " in L'interprétation de la Convention européenne des droits de l'homme, Bruxelles, Bruylant, 1998, p. 93-131.

TERRIS, D. et al, The International Judge, Oxford, Oxford University Press, 2007, p. 71-79.

WESTON, M., "Characteristics and constraints of producing bilingual judgments: the example of the European Court of Human Rights " in GEMAR and KASIRER (dir.), Jurilinguistique : entre langues et droits, Bruxelles, Bruylant, 2005, p. 445-459. 